\title{
Habilidades sociais em portadores de câncer de estômago
}

\author{
Social skills in stomach cancer bearers
}

\author{
Taísa Borges GRÜN
}

\begin{abstract}
Resumo
O objetivo deste estudo foi detectar possíveis déficits em habilidades sociais, por meio do Inventário de Habilidades Sociais em pacientes com câncer de estômago em um hospital de tratamento do câncer em Curitiba. Participaram deste estudo 30 adultos de ambos os sexos. Os resultados revelaram que as mulheres participantes da pesquisa apresentaram um repertório de habilidades sociais mediano, enquanto os homens participantes da pesquisa apresentaram um déficit em habilidades sociais. Os participantes com escolaridade de segundo grau apresentaram uma média superior aos participantes com escolaridade entre primeira a quarta série para um dos fatores analisados pelo inventário. Não houve diferença estatisticamente significativa entre os participantes da pesquisa de acordo com a idade. Como os resultados obtidos no presente estudo não são conclusivos, sugere-se a replicação da presente pesquisa, tomando esses achados como ponto de partida.
\end{abstract}

Palavras-chave: habilidades sociais; neoplasias gástricas; prevenção.

\begin{abstract}
The objective of this study was to detect possible social skills deficits, through Inventário de Habilidades Sociais (Del Prette \& Del Prette, 2001) application in stomach cancer patients from a hospital of cancer treatment in Curitiba, PR, Brazil. Thirty adults from both sexes had participated in this study. According to the outcomes, women presented social skills medium repertory, while men presented social skills deficit. The participants from highschool presented superior average than the participants from the elementary school in one of the inventory factors. There was no age statistical significant difference. As these results are not conclusive, the present research replication is suggested, taking these discoveries as a starting point.
\end{abstract}

Key words: social skills; stomach neoplasms; prevention.

O câncer gástrico é a segunda causa de morte por neoplasia no mundo, ocorrendo com grande freqüência no Japão e na China. Os homens são mais acometidos, numa proporção de 1,7:1 em relação às mulheres; mas a mortalidade é semelhante nos dois sexos. A incidência aumenta com a idade e a etnia negra é 1,5 vez mais acometida (Schwartsmann, Mattei \& Moreira, 2004). Algumas pesquisas também apontam que o câncer gástrico é mais comum em classes socioeconômicas mais baixas (Bernhard \& Hürny, 1998; Hanks, Jones \& Minasi, 1996). Segundo pesquisa realizada no Havaí numa sociedade urbana, existe diferença na prevalência de determinados tipos de carcinoma gástrico de acordo com o sexo e a idade dos participantes, bem como quanto à localização do carcinoma no estômago de acordo com a etnia

\section{V V V}

1 Estagiária, Serviço de Psicologia Clínica, Hospital Erasto Gaertner. Rua Dr. Ovande do Amaral, 201, Jardim das Américas, 81520-060, Curitiba, PR, Brasil. E-mail: <taisabg@hotmail.com>.

Agradecimentos à instituição que possibilitou a realização desta pesquisa e a todos que de alguma forma contribuíram para sua concretização. 
(Stemmerman, Nomura, Kolonel, Goodman \& Wilkens, 2002). No Brasil e em outros países da América Latina a incidência de câncer de estômago vem aumentando (Montagnini, Anelli, Costa \& Valadares, 2002).

Segundo um levantamento estatístico realizado pelo hospital de tratamento de câncer participante da presente pesquisa (LPCC, 2003), entre 1990 e 1999, foram registrados 659 casos de câncer de estômago, o que corresponde a 3,1\% dos pacientes atendidos pelo hospital nesse período, sendo a sétima topografia mais freqüente. A distribuição entre os sexos foi de aproximadamente 3:1, sendo 477 pacientes do sexo masculino - correspondente a 5,0\% dos pacientes homens atendidos - e 182 do sexo feminino - correspondente a 1,6\% das pacientes mulheres atendidas. 0 tumor de estômago foi mais diagnosticado em pacientes a partir de 50 anos, com raros casos abaixo dos 30 anos e acima dos 80 anos. Nesse período, 57,7\% dos casos eram casos avançados da doença e 16,4\% dos casos tinham doença inicial.

Geralmente o diagnóstico do câncer gástrico é feito tardiamente, nos estágios avançados, o que dificulta o tratamento curativo. Isso se deve, em parte, aos sinais e sintomas serem inespecíficos, incluindo dor, perda de peso, anorexia, náuseas, vômitos e hematêmese em alguns casos (Hanks et al., 1996; Schwartsmann et al., 2004). Reis (2004) afirma que o fato de o diagnóstico freqüentemente ocorrer em fases avançadas da doença traz como conseqüência uma sobrevida de cinco anos para apenas 5\%-15\% dos pacientes.

A etiologia do câncer gástrico parece estar relacionada a vários fatores: predisposição genética, infecções e componentes da dieta, mas o papel do tabaco e do álcool como fatores de risco ainda se mostraram inconsistentes (Schwartsmann et al., 2004). Montagnini et al. (2002) também destacam a radiação, câncer de colo hereditário não associado a polipose, metaplasia intestinal, anemia perniciosa e síndrome de Li-Fraumeni como fatores associados para o aumento desse tipo de câncer. De forma geral, as condições gastrintestinais vêm sendo associadas à presença de transtornos mentais ou conflitos psicológicos em sua etiologia e curso (Mello Filho, 1992; Neves Neto, 2001; Pacheco \& Silva, 1962).

Considerando-se que fatores psicossociais e comportamentais - entre eles o estilo de vida, envolvimento social, papéis sociais, personalidade, estilo de enfrentamento (coping) e estados emocionais - alteram o risco para o câncer e sua sobrevivência (Fox, 1998; Helgson, Cohen \& Fritz, 1998; Holland, 1990; Watson \& Greer, 1998), percebe-se a necessidade de mais pesquisas no sentido de caracterizar o repertório de comportamentos de pacientes portadores de câncer. Dentro desse repertório mais amplo encontra-se o repertório de habilidades sociais. Durante atendimentos psicológicos realizados com pacientes com câncer de estômago percebeu-se que muitos deles tinham dificuldades em seus relacionamentos pessoais, bem como falta de assertividade no que se refere ao seu posicionamento frente à equipe médica do hospital participante da pesquisa. Essa experiência trouxe à tona o questionamento que deu origem à presente pesquisa: seria o déficit em habilidades sociais uma constante entre os pacientes com câncer de estômago?

Atualmente, o conceito de habilidades sociais é visto de maneira mais ampla, englobando não só assertividade, mas também empatia, solução de problemas e leitura do ambiente: "Habilidades Sociais correspondem a um universo mais abrangente das relações interpessoais e se estendem para além da assertividade, incluindo as habilidades de comunicação, de resolução de problemas, de cooperação e aquelas próprias dos rituais sociais estabelecidos pela subcultura geral" (Del Prette \& Del Prette; 1999, p.29).

Del Prette e Del Prette (2001a, 2001 b) propõem uma organização das principais classes e subclasses de habilidades sociais. Além da reação assertiva de enfrentamento - que é definida como "o exercício dos próprios direitos e a expressão de qualquer sentimento, com controle da ansiedade e sem ferir os direitos do outro ..., podendo, portanto, ser caracterizada como reação de enfrentamento" (Del Prette \& Del Prette, 2001a, p.12), os autores referem as habilidades sociais de comunicação (fazer e responder a perguntas, pedir feedback, gratificar/elogiar, dar feedback, iniciar, manter e encerrar conversação), habilidades sociais de civilidade (dizer por favor, agradecer, apresentar-se, cumprimentar, despedir-se), habilidades sociais assertivas, direito e cidadania (manifestar opinião, concordar, discordar, fazer, aceitar e recusar pedidos, desculpar-se, admitir falhas, interagir com autoridade, estabelecer relacionamento afetivo e/ou sexual, encerrar relacionamento, expressar 
raiva/desagrado, pedir mudança de comportamento e lidar com críticas), habilidades sociais empáticas (parafrasear, refletir sentimentos, expressar apoio), habilidades sociais de trabalho (coordenar grupo, falar em público, resolver problemas, tomar decisões e mediar conflitos, habilidades sociais educativas) e habilidades sociais de sentimento positivo (fazer amizade, expressar solidariedade, cultivar o amor).

Diferentes contextos contribuem para a aprendizagem de desempenhos sociais, os quais dependem de um repertório de habilidades sociais, uma vez que geram demandas diferentes e o exercício de leitura do ambiente (Löhr \& Grün, 2004). Segundo Dell Prette e Dell Prette (2001b), a identificação ou decodificação das demandas para um desempenho social depende da leitura crítica do ambiente social.

As habilidades sociais têm sido relacionadas à melhor qualidade de vida, a relações interpessoais mais gratificantes, à maior realização pessoal e ao sucesso profissional (Caballo, 1987, 1991; Collins \& Collins, 1992). Del Prette e Del Prette (2001a) colocam que pesquisas realizadas no campo de treinamento em habilidades sociais indicam que pessoas socialmente competentes apresentam relações pessoais e profissionais mais produtivas, satisfatórias e duradouras, além de melhor saúde física e mental. Por outro lado, déficits em habilidades sociais estão associados a dificuldades e conflitos nas relações interpessoais, a uma pior qualidade de vida e a diversos transtornos psicológicos como a timidez, o isolamento social, a delinqüência juvenil, o desajustamento escolar, o suicídio e os problemas conjugais, bem como síndromes como depressão, pânico social e esquizofrenia (Del Prette \& Del Prette, 2001a).

Segundo Del Prette e Del Prette (1999, 2001 b), os déficits em habilidades sociais podem se refletir, até certo ponto, em uma freqüência muito baixa ou nula para determinados comportamentos, o que pode estar associado à dificuldade ou ansiedade em emitir esse desempenho. Assim, pode-se esperar que dificuldade e freqüência tenham uma correlação negativa entre si no que diz respeito às habilidades sociais (Del Prette \& Del Prette, 2002). Segundo dados estatísticos do próprio hospital participante da pesquisa, os pacientes com câncer de estômago que freqüentam esse hospital estão próximos da faixa etária do idoso, o que pode significar a dificuldade em conquistar e manter redes de apoio social e garantir melhor qualidade de vida (Carneiro \& Falcone, 2004).

O possível papel dos fatores psicológicos no risco do câncer e sobrevida tem recebido considerável atenção nos últimos trinta anos (Garssen, 2004). Muito desse interesse deriva de algumas hipóteses largamente difundidas. A primeira hipótese diz respeito à existência de uma base biológica comum, que determina a personalidade e a resposta psicológica ao estresse, bem como o risco para o desenvolvimento do câncer. A segunda hipótese é aquela que afirma que a personalidade predispõe a certos comportamentos e expõe a fatores de estilo de vida, como fumar e certos padrões de alimentação, os quais influenciam o risco para o câncer. Outra possibilidade de explicação é aquela em que fatores psicológicos podem prejudicar a função imune, que poderia predispor o organismo ao início e à progressão de uma neoplasia (Dalton, Boesen, Ross, Schapiro \& Johansen, 2001; Schapiro, Nielsen, Jorgensen, Boesen \& Johansen, 2002).

Assim, surgiram diversos estudos com o objetivo de verificar essas hipóteses. Algumas pesquisas têm sido feitas a fim de verificar se algumas características da personalidade estão mais associadas ao risco para o câncer (Hansen, Floderus, Frederiksen \& Johansen, 2005; Nakaya et al., 2003; Schapiro, Nielsen, Jorgensen, Boesen \& Johansen, 2002; Schapiro et al., 2001; Watson \& Greer, 1998). Outras pesquisas buscaram verificar relações entre papéis sociais e câncer (Helgson et al., 1998), fatores psicossociais e câncer (Fox, 1998; Holland, 1990), e psiconeuroimunologia e câncer (Bovbjerg \& Valdimarsdottir, 1998).

Finalmente, existem pesquisas com o objetivo de fazer um levantamento sobre os estudos realizados nessas áreas, bem como sobre os resultados encontrados (Dalton et al., 2001; Garssen, 2004). Entretanto, as evidências quanto ao papel dos fatores psicológicos no surgimento e progressão do câncer ainda não são muito claras. Infelizmente, a maior parte dos estudos realizados apresenta problemas metodológicos, o que compromete os resultados alcançados (Dalton et al., 2001; Garssen, 2004; Hansen et al., 2005). 
Garssen (2004) afirma que, apesar de os resultados esperados não terem sido consistentemente demonstrados em uma série de estudos, algumas tendências importantes emergiram. A repressão de sentimentos negativos, o comportamento de minimizar o impacto da doença e o sentimento de incapacidade (helplessness) parecem estar associados à progressão da doença, bem como suporte social, eventos de perda e estado civil (Garssen, 2004). Também foram encontrados resultados interessantes no que diz respeito a um tipo de personalidade-caracterizado por comportamentos como falta de assertividade, não expressão de sentimentos negativos (especialmente a raiva) e submissão social (social compliance) - mais freqüentemente presente em pessoas que desenvolvem câncer, mas não existem claras evidências de uma relação causal entre esse tipo de personalidade e o câncer (Watson \& Greer, 1998). Da mesma forma, grande parte da literatura sugere que a integração social - como, por exemplo, a freqüência de contatos sociais - está associada a baixos índices de mortalidade e melhor ajustamento à doença (Fox, 1998; Helgson et al., 1998).

Apesar dessas dificuldades, Garssen (2004) afirma que existe a possibilidade de os fatores psicológicos, os quais não apresentaram até o momento um fator preditivo para o câncer quando estudados isoladamente, tenham um efeito na interação com fatores demográficos e médicos.

Independentemente do fato de certas características de personalidade ou certos fatores psicossociais estarem associados ou não ao risco para o desenvolvimento de câncer, a caracterização do repertório de habilidades sociais de pacientes portadores de câncer de estômago faz-se importante, uma vez que pode contribuir para o melhor entendimento do repertório comportamental desses pacientes.

Isto posto, o objetivo desta pesquisa foi caracterizar uma amostra de portadores de câncer de estômago de um hospital de tratamento do câncer em Curitiba, segundo a variável habilidades sociais, por meio do Inventário de Habilidades Sociais (IHS) (Del Prette \& das habilidades sociais (tais como habilidades sociais de enfrentamento e auto-afirmação com risco,

154 auto-afirmação na expressão de sentimento positivo, conversação e desenvoltura social, auto-exposição a desconhecidos e situações novas, e autocontrole da agressividade) nos pacientes portadores de câncer de estômago de um hospital de tratamento do câncer em Curitiba.

\section{Método}

\section{Participantes}

Trinta pacientes de um hospital de tratamento do câncer em Curitiba diagnosticados com câncer de estômago. Participaram da pesquisa 9 mulheres e 21 homens. Quanto à escolaridade, um participante era analfabeto, 16 encontravam-se entre a $1^{\text {a }}$ e a $4^{\mathrm{a}}$ séries do ensino fundamental, 9 encontravam-se entre a $5^{\text {a }}$ e a $8^{a}$ séries do ensino fundamental e 4 encontravam-se entre as séries do $2^{\circ}$ grau. Quanto à idade, 6 participantes tinham idade menor ou igual a 39 anos, 10 participantes entre 40 e 49 anos, 8 entre 50 e 59 anos e 6 participantes tinham idade igual ou maior a 60 anos.

Os participantes foram distribuídos em grupos para facilitar as análises estatísticas: A) de acordo com a idade e B) de acordo com a escolaridade. De acordo com a idade, os participantes foram divididos em: (1) idade menor ou igual a 39 anos, (2) entre 40 e 49 anos, (3) entre 50 e 59 anos e (4) idade igual ou maior a 60 anos. De acordo com a escolaridade os participantes foram divididos em: (1) analfabetos, (2) $1^{\mathrm{a}}$ a $4^{\mathrm{a}}$ séries, (3) $5^{\mathrm{a}}$ a $8^{\mathrm{a}}$ séries e (4) $2^{\circ}$ grau.

\section{Instrumento}

O instrumento foi aplicado no ambulatório do Serviço de Psicologia Clínica e nas demais dependências de um hospital de tratamento do câncer em Curitiba, entre janeiro e abril de 2005.

O Inventário de Habilidades Sociais (IHS) (Del Prette \& Del Prette, 2001) é composto por 38 questões distribuídas entre diversas habilidades sociais: fazer elogios, responder a elogios, recusar pedidos irrazoáveis, expressar discordância, manejar crítica/expressar desagrado, solicitar mudança de comportamento, defender direitos próprios e de outrem, expressar sentimento positivo, pedir favores, fazer perguntas, cumprimentar/iniciar conversação, participar e man- 
ter conversação, encerrar conversação, falar em público.

Essas habilidades estão agrupadas no IHS em cinco fatores: F1 (enfrentamento e auto-afirmação com risco), F2 (auto-afirmação na expressão de sentimento positivo), F3 (conversação e desenvoltura social), F4 (auto-exposição a desconhecidos e situações novas) e F5 (autocontrole da agressividade).

\section{Procedimento}

O contato inicial foi feito em quatro situações: 1) paciente diagnosticado com câncer de estômago encaminhado a uma avaliação pré-operatória do serviço de psicologia do hospital; 2) paciente diagnosticado com câncer de estômago internado para procedimento cirúrgico no hospital; 3) paciente com consulta agendada (revisão) no ambulatório de cirurgia abdominal; 4) paciente em tratamento quimioterápico no hospital.

Os participantes preencheram um termo de consentimento para a participação na pesquisa, bem como da utilização dos dados coletados (anonimamente) em publicações.

Após o preenchimento do termo de consentimento, foi feita a aplicação do IHS, individualmente, no ambulatório de psicologia (em caso de paciente encaminhado para avaliação pré-operatória ou revisão) ou na ala em que o paciente estava internado. Ao contrário do indicado pelos autores no manual de aplicação, o inventário foi aplicado em adultos com baixo nível de escolaridade, correspondente à maior parte da população do hospital participante da pesquisa. A pesquisadora optou por esse instrumento devido à escassez de inventários de larga utilização ou validados que mensurem as habilidades sociais. Com o objetivo de facilitar o entendimento e preenchimento do inventário pelos participantes da pesquisa, a pesquisadora o leu em voz alta.
Os dados computados com o IHS foram analisados por meio do programa estatístico SPSS 10.0.

\section{Resultados}

Comparando-se a média dos escores totais do sexo feminino (Tabela 1) com os dados normativos do grupo amostral do IHS (Del Prette \& Del Prette, 2001), pode-se observar que a média dos escores totais das participantes da pesquisa são referentes a um repertório de habilidades sociais ligeiramente abaixo da média do grupo amostral (sexo feminino) do inventário (ET=92). Já comparando a média dos escores totais do sexo masculino com os dados normativos do grupo amostral (sexo masculino) (ET=96) do IHS (Del Prette \& Del Prette, 2001), observa-se que os homens participantes da presente pesquisa apresentam, na média dos escores totais, um baixo repertório de habilidades sociais, com indicação para treinamento em habilidades sociais (ET $\leq 88,5)$.

Com relação aos escores fatoriais do IHS para o sexo feminino, comparando-se as médias obtidas (Tabela 1) com os dados normativos do grupo amostral (sexo feminino) do inventário ( $F 1=8,4 ; F 2=9,62$; $F 3=6,59 ; F 4=3,32 ; F 5=1,15)$, percebe-se que para os escores F1 (enfrentamento e auto-afirmação com risco) e F2 (auto-afirmação na expressão de sentimento positivo), as participantes da pesquisa apresentam um repertório de habilidades sociais ligeiramente acima da média para esses dois fatores. Fazendo-se a mesma comparação com os fatores F3 (conversação e desenvoltura social) e F4 (auto-exposição a desconhecidos e situações novas), encontra-se que as participantes da pesquisa apresentam um repertório de habilidades sociais ligeiramente abaixo da média para esses dois fatores. Já a média para o fator F5 (autocontrole da

Tabela 1. Média e desvio-padrão da idade, escore total e escores fatoriais dos participantes da pesquisa divididos por sexo.

\begin{tabular}{|c|c|c|c|c|c|c|c|c|}
\hline Sexo & & Idade (anos) & ET & F1 & $\mathrm{F} 2$ & F3 & $\mathrm{F} 4$ & F5 \\
\hline \multirow[t]{2}{*}{ Feminino $(n=9)$} & Média & 44,78 & 90,00 & 8,64 & 9,99 & 6,05 & 2,76 & 0,38 \\
\hline & Desvio-padrão & 10,51 & 14,47 & 2,91 & 1,15 & 2,12 & 1,10 & 0,97 \\
\hline \multirow[t]{2}{*}{ Masculino $(n=21)$} & Média & 52,52 & 85,61 & 8,59 & 8,93 & 6,25 & 2,39 & 1,41 \\
\hline & Desvio-padrão & 12,66 & 23,95 & 4,00 & 1,80 & 2,89 & 1,7 & 0,63 \\
\hline
\end{tabular}


agressividade) da Tabela 1 demonstra um baixo repertório de habilidades sociais das participantes para esse fator, com indicação para treinamento em habilidades sociais (F5 $\leq 0,42)$.

Quanto às médias dos escores fatoriais apresentados na Tabela 1 para o sexo masculino, quando comparadas aos dados normativos do grupo amostral (sexo masculino) do IHS (F1= 10,35; F2= 8,79; F3=6,72; $F 4=3,3 ; F 5=1,41$ ), observa-se que para os escores $F 1$ (enfrentamento e auto-afirmação com risco) e F4 (auto-exposição a desconhecidos e situações novas), os homens participantes da pesquisa apresentam um baixo repertório de habilidades sociais para esses fatores, com indicação de treinamento em habilidades sociais ( $F 1 \leq 8,89 ; F 4 \leq 2,49)$. Fazendo-se a mesma comparação para os demais fatores apresentados na Tabela 1, encontra-se que os homens participantes da pesquisa apresentam um repertório de habilidades sociais próximo da média do grupo amostral (sexo masculino).

Novamente é importante ressaltar que, apesar da ressalva dos autores do IHS (Del Prette \& Del Prette, 2001) com relação ao nível de escolaridade para a utilização do instrumento (que corresponde à formação de segundo grau), esse foi utilizado na presente pesquisa devido à inexistência de inventários de larga utilização ou validados que mensurem as habilidades sociais. Assim, os dados obtidos devem ser observados com cautela.

Ainda é importante ressaltar que os desvios-padrão apresentados na Tabela 1 foram bastante elevados para alguns itens (idade e escore total) tanto para o sexo feminino quanto para o sexo masculino.

Com o objetivo de verificar possíveis diferenças estatisticamente significativas na média dos resultados obtidos na pesquisa de acordo com o sexo dos participantes, utilizou-se o teste não paramétrico $U$ de Mann-Whitney, aceitando como significativas diferenças com $p \leq 0,05$. Esse teste revelou que existe diferença estatisticamente significativa entre os resultados obtidos no escore fatorial F5 (autocontrole da agressividade) de acordo com o sexo do participante da pesquisa $\rightarrow \quad(U=32,5 ; p=0,004)$, sendo a média masculina $(1,41)$ 足: superior à feminina $(0,38)$ para esse fator.

Em seguida, para verificar diferenças estatisticamente significativas na média dos resultados obtidos na pesquisa de acordo com a escolaridade dos partici- pantes, utilizou-se o teste estatístico não paramétrico Kruskal-Wallis, aceitando como significativas diferenças com $p \leq 0,05$. A análise estatística revelou haver diferença estatisticamente significativa para o escore fatorial F2 (auto-afirmação na expressão de sentimento positivo) e para o escore total ET. Visto que o teste estatístico não paramétrico Kruskal-Wallis dá a diferença geral, mas não aponta entre quais grupos essas diferenças especificamente ocorreram, utilizou-se o teste não paramétrico U de Mann-Whitney, que demonstrou haver diferença nas médias do fator F2 entre os grupos 2 ( 1 a a $4^{\text {a }}$ séries) e 4 ( $\left.2^{\circ} \mathrm{grau}\right)\left(\chi^{2}=9,111 ; \mathrm{gl}=3 ; p=0,016\right)$, sendo a média do grupo $4(16,63)$ superior à do grupo $2(8,97)$. Para o escore total ET, o teste não paramétrico $U$ de Mann-Whitney demonstrou haver diferenças estatisticamente significativas entre as médias do grupo 2 (1ª a $4^{\text {a }}$ séries; média 10,41) e 3 (5a a 8a séries; média $17,61)\left(\chi^{2}=8,863 ; g l=3 ; p=0,017\right)$, e entre os grupos 2 ( 1 a a $4^{\text {a }}$ séries; média 9,06) e 4 ( $2^{\circ}$ grau; média 16,25$)\left(\chi^{2}=8,863\right.$; $g l=3 ; p=0,029)$.

Finalmente, para verificar diferenças estatisticamente significativas na média dos resultados obtidos na pesquisa de acordo com os grupos de idade dos participantes, utilizou-se novamente o teste KruskalWallis, aceitando como significativas diferenças com $p \leq 0,05$. Esse teste revelou que não existe diferença estatisticamente significativa nos resultados obtidos no IHS de acordo com o grupo de idade do participante.

\section{Discussão}

Analisando-se a idade dos participantes da amostra da presente pesquisa, percebe-se que esses estão na faixa etária entre 40 e 60 anos, um pouco abaixo dos pacientes do mesmo hospital que foram tratados de câncer gástrico entre 1990 e 1999 (LPCC, 2003).

Quanto aos resultados obtidos pelas mulheres participantes da pesquisa a partir da aplicação do IHS (Del Prette \& Del Prette, 2001), chama a atenção a baixa média obtida pelas mulheres participantes da pesquisa para o fator F5 (autocontrole da agressividade), apresentando diferença estatisticamente significativa quando comparada com a média obtida pelos homens para esse fator. Esse resultado apresenta indicação para treino em habilidades sociais (Del Prette \& Del Prette, 2001) e pode estar relacionado ao que os participantes consi- 
deraram comportamento agressivo durante a aplicação do inventário: muitas mulheres referiram comportamento agressivo como broncas e gritos, enquanto muitos homens referiram-no como bater.

Já comparando a média dos escores totais e fatoriais do sexo masculino com os dados normativos do grupo amostral (sexo masculino) do IHS (Del Prette \& Del Prette, 2001), observa-se que tanto a média dos escores totais dos homens participantes da presente pesquisa quanto a média dos escores F1 (enfrentamento e auto-afirmação com risco) e F4 (auto-exposição a desconhecidos e situações novas) apresentam-se bem abaixo da média, isto é, os homens participantes da pesquisa apresentam um baixo repertório de habilidades sociais, com indicação de treinamento em habilidades sociais. Esses baixos índices de repertório em habilidades sociais podem se refletir em conquistar e manter redes de apoio social (Carneiro \& Falcone, 2004), em dificuldades referentes à assertividade e expressão de sentimentos negativos (Garssen, 2004), bem como em dificuldades no enfrentamento da doença. Infelizmente esses fatores são indicativos de um prognóstico ruim em muitas pesquisas (Fox, 1998; Garssen, 2004; Helgson et al., 1998; Watson \& Greer, 1998), sendo indicada intervenção para minimizar o possível efeito de tais déficits para esses pacientes.

Outro dado relevante diz respeito aos resultados obtidos quanto à escolaridade dos participantes. Os testes estatísticos revelaram que existe diferença estatisticamente significativa para o escore fatorial F2 (auto-afirmação na expressão de sentimento positivo) de acordo com a escolaridade do participante, sendo a média do grupo 4 ( $2^{\circ}$ grau) superior à do grupo 2 ( $1^{\text {a a }} 4^{\mathrm{a}}$ séries). Também revelaram que existe diferença estatisticamente significativa para o escore total ET entre os grupos 2 ( $1^{\text {a a }} 4^{\mathrm{a}}$ séries) e 3 ( $5^{\mathrm{a}}$ a $8^{\mathrm{a}}$ séries) e entre os grupos 2 ( $1^{\text {a a }} 4^{\text {a }}$ séries) e 4 ( $2^{\circ} \mathrm{grau}$ ), sendo maiores as médias dos grupos de maior escolaridade. Esse resultado pode estar associado ao fato do IHS ser indicado para pessoas com escolaridade mínima de $2^{\circ}$ grau (Del Prette \& Del Prette, 2001), o que talvez tenha dificultado a compreensão dos participantes de escolaridade mais baixa nas questões referentes a este fator. Entretanto, isso também pode indicar que o aprendizado das habilidades sociais está associado, de alguma forma, à escolaridade, fazendo com que pessoas de maior escolaridade tenham essa habilidade mais desenvolvida. Novas pesquisas podem colaborar na compreensão dessas questões.

Finalmente, é importante ressaltar que, apesar de os dados obtidos com a presente pesquisa não serem conclusivos devido à pequena amostra e ao instrumento utilizado, eles indicam a probabilidade de existir realmente um déficit em habilidades sociais em pacientes com câncer de estômago, principalmente em homens.

Assim, propõe-se a replicação desta pesquisa em situações futuras, com a utilização de uma amostra mais ampla, bem como a utilização de outros instrumentos além do IHS (Del Prette \& Del Prette, 2001), podendo-se analisar além do repertório de habilidades sociais dos pacientes, comportamentos referentes à saúde, características demográficas e médicas. Sugerem-se também a identificação do tipo histopatológico de câncer gástrico e a diferenciação da amostra entre pacientes que ainda não receberam o diagnóstico e pacientes que já têm conhecimento da doença, com o objetivo de obter o maior número de dados possíveis sobre o paciente e a doença.

Os dados obtidos não são conclusivos, mas indicam a probabilidade de existir um déficit em habilidades sociais em pacientes com câncer de estômago.

\section{Referências}

Bernhard, J., \& Hürny, C. (1998). Gastrointestinal cancer. In J. C. Holland (Org.), Psycho-oncology (pp.324-339). New York: Oxford University Press.

Bovbjerg, D. H., \& Valdimarsdottir, H. B. (1998). Psychoneuroimmunology: implications for Psycho-oncology. In J. C. Holland (Org.), Psycho-oncology (pp.125-134). New York: Oxford University Press.

Caballo, V. E. (1987). Teoria, evaluoción yentrenamiento de las habilidades sociales. Valencia: Promolibro.

Caballo, V. E. (1991). El entrenamiento en habilidades sociales. In V. E. Caballo (Org.), Manual de técnicas de terapiay modificación de conducta. Madrid: Siglo Veintiuno,

Carneiro, R. S., \& Falcone, E. M. O. (2004). Um estudo das capacidades e deficiências em habilidades sociais na terceira idade. Psicologia em Estudo, 9 (1), 119-126.

Collins, J., \& Collins, M. (1992). Social Skills training and the professional helper. New York: Willey. 
Dalton, S. O., Boesen, E. H., Ross, L., Schapiro, I. R., \& Johansen, C. (2001). Mind and cancer: do psychological factors cause cancer? European Journal of Cancer, 38 (1), 1313-1323.

Del Prette, Z. A. P., \& Del Prette, A. (1999). Psicologia das habilidades sociais. Petrópolis: Vozes.

Del Prette, Z. A. P., \& Del Prette, A. (2001a). Inventário de habilidades sociais: manual de aplicação, apuração e interpretação. São Paulo: Casa do Psicólogo.

Del Prette, Z. A. P., \& Del Prette, A. (2001b). Psicologia das relações interpessoais: vivências para o trabalho em grupo. Petrópolis: Vozes.

Del Prette, Z. A. P., \& Del Prette, A. (2002). Avaliação de habilidades sociais de crianças com um inventário multimídia: indicadores sociométricos associados à freqüência versus dificuldade. Psicologia em Estudo, 7 (1), 61-73.

Engstrom, P. F. (2004). Cancer prevention: the future for oncology. Acta Oncológica Brasileira, 24 (1), 586-600.

Fox, B. H. (1998). Psychosocial factors in cancer incidence and prognosis. In J. C. Holland (Org.), Psycho-oncology (pp.110-124). New York: Oxford University Press.

Garssen, B. (2004). Psychological factors and cancer development: evidence after 30 years of research. Clinical Psychology Review, 24 (1), 315-338.

Hanks, J. F., Jones, R. S., \& Minasi, J. S. (1996). Tumors of the stomach and duodenum. In G. D. Zuidema. Shackelford's surgery of the aliment tract. Philadelphia: W. B. Saunders.

Hansen, P. E., Floderus, B., Frederiksen, K., \& Johansen, C. (2005). Personality traits, health behavior, and risk for cancer. Cancer, 103 (5), 1082-1091.

Helgson, V. S., Cohen, S., \& Fritz, H. L. (1998). Social ties and cancer. In J. C. Holland (Org.), Psycho-oncology (pp.99-109). New York: Oxford University Press.

Holland, J. C. (1990). Gastrointestinal cancer. In J. C. Holland J. H. Rowland (Orgs.), Handbook of Psychooncology (pp.208-217). New York: Oxford University Press.

Holland, J. C. (1990). Behavioral and psychosocial risk factors in cancer: human studies. In J. C. Holland \& J. H. Rowland (Orgs.), Handbook of Psychooncology (pp.705-726). New York: Oxford University Press.

Liga Paranaense de Combate ao Câncer. (2003). 10 Anos de registro hospitalar de câncer. Curitiba: LPCC.
Löhr, S. S., \& Grün, T. B. (2004). Desenvolvendo habilidades pró-sociais: grupo de crianças e grupo de pais. Relatório do UFPR/TN, não-publicado. Curitiba: UFPR.

Mello Filho, J. (1992). Psicossomática hoje. Porto Alegre: Artes Médicas.

Montagnini, A. L., Anelli, A., Costa, M. L. V., \& Valadares, A. D. (2002). Câncer de estômago. In L. P. Kowalski, A. Anelli, J. V. Salvajoli \& L. F. Lopes (Orgs.), Manual de condutas diagnósticas e terapêuticas em oncologia. São Paulo: Âmbito Editores.

Nakaya, N., Tsubono, Y., Hosokawa, T., Nishino, Y., Ohkubo, T., Hozawa, A., Shibuya, D., Fukudo, S., Fukao, A., Tsuji, I., \& Hisamichi, S. (2003). Personality and the risk of cancer. Journal of the National Cancer Institute, 95 (11), 799-805.

Neves Neto, A. R. (2001). Aplicação da Terapia Cognitivo-Comportamental para doenças gastrintestinais funcionais. In H. J. Guilhardi, M. B. B. P. Madi, P. P. Queiroz \& M. C. C. Scoz (Orgs.), Sobre comportamento e cognição (Vol. 8). Santo André: Esetec.

Pacheco e Silva, A. C. (1962). Medicina psicossomática. São Paulo: Serviço de Documentação Científica.

Reis, L. F. L. (2004). Molecular classifiers for gastric cancer and non-malignant diseases of the gastric mucosa. Acta Oncológica Brasileira, 24 (1), 576-579.

Schapiro, I. R., Ross-Petersen, L., Saelen, H., Garde, K., Olsen, J. H., \& Johansen, C. (2001). Extroversion and Neuroticism and the Associated Risk of Cancer: a Danish Cohort Study. American Journal of Epidemiology, 153 (8), 757-763.

Schapiro, I. R., Nielsen, L. F., Jorgensen, T., Boesen, E. H., \& Johansen, C. (2002). Psychic Vulnerability and the associated risk for cancer. Cancer, 94 (12), 3299-3306.

Schwartsmann, G., Mattei, J., \& Moreira, L. F. (2004). Neoplasias gastrintestinais. In J. R. Q. Guimarães (Org.), Manual de oncologia. São Paulo: BBS Editora.

Stemmermann, F. N., Nomura, A. M. Y., Kolonel, L. N., Goodman, M. T., \& Wilkens, L. R. (2002). Gastric carcinoma: pathology findings in a multiethnic population. Cancer, 95 (4), 744-750.

Watson, M., \& Greer, S. (1998). Personality and Coping. In J. C. Holland (Org.), Psycho-oncology (pp.91-98). New York: Oxford University Press.

Recebido em: 27/6/2005

Versão final reapresentada em: 7/11/2005

Aprovado em: 22/11/2005 